\title{
Propuesta para el diseño de rutas turísticas culturales "El caso del sur del estado de Jalisco, México"
}

\section{Proposal for designing cultural touristic routes "The case of the southern region in the state of Jalisco, Mexico"}

\author{
Pablo Noé Arredondo Ochoa (ARREDONDO OCHOA, P. N.) ${ }^{*}$ \\ Carlos Hernández Vega (HERNÁNDEZ VEGA, C.) ${ }^{* *}$ \\ Tania Delfina Mendoza Tolentino (MENDOZA TOLENTINO, T. D.) ${ }^{* * *}$
}

\begin{abstract}
RESUMEN - El presente trabajo es uno de los resultados de una tesis de especialidad en turismo, donde se presenta una propuesta para la valoración del patrimonio cultural con el fin de crear rutas de turismo cultural, caso de la región sur del estado de Jalisco, México. Se analizan los factores de accesibilidad y disponibilidad para poder hacer de un patrimonio un destino turístico, así como, los factores que se deben tomar en cuenta para decidir los atractivos a incluir en la ruta, sus características, los servicios que coadyuvan en la elaboración de un producto turístico competitivo (hospedaje, alimentación, transporte y servicios complementarios). También se incluye sugerencias para la capacitación a los prestadores de servicios turísticos (PST), la señalización de atractivos, distancias y sitios, a su vez la comercialización de la ruta como producto turístico, es decir, las estrategias que se recomienda utilizar para posicionar la ruta de turismo cultural, quienes son los evaluadores de la ruta turística y por ultimo dónde empezar y dónde concluir la ruta de acuerdo a la disponibilidad de tiempo y la disponibilidad económica del turista.
\end{abstract}

Palabras Clave: Patrimonio turístico cultural; Diseño de rutas; Competitividad; Sur del estado de Jalisco.

ABSTRACT - This paper is a result of a master degree thesis in Tourism, which is a proposal for the valuation of cultural heritage in order to create cultural tourism routes, in the southern region of the state of Jalisco, Mexico. The factors of accessibility and availability are analyzed to do a heritage tourist destination, as well as the factors to be taken into account in deciding the attractions included in the route, its features, services that assist in the development of competitive tourism product (lodging, food, transport and complementary services). It also includes suggestions for training the tourism service providers (TSP), attractive signaling, distances and locations, also the marketing of the route as a tourism product, and also the strategies recommended to position the

\footnotetext{
* Estudiante de la Maestría en Competitividad Turística (Universidad de Colima). Avenida Josefa Ortiz de Domínguez \# 64, Villa de Álvarez, Colima. México. Teléfono +52 (312) 3161182 E-mail: pablo_arredondo.lic_turismo@hotmail.com.

** Doctor en Ciencias para el Desarrollo Sustentable (Universidad de Guadalajara). Profesor Titular del Departamento de Desarrollo Regional del Centro Universitario del Sur. Av. Enrique Arreola Silva No. 883, colonia centro C. P. 49000, Ciudad Guzmán, Jalisco, México. Teléfono: +52 (341) 575 2222, Fax: 01 (341) 5752223. E-mail: caheve2020@ hotmail.com / chhernand@cusur.udg.mx

**** Especialidad en Dirección de Organizaciones Turísticas. (Universidad de Colima), México. Subdirectora de la empresa de capacitación en turismo CAVITUR. Marcos Gordoa 282, Colonia Centro, Ciudad Guzmán Jalisco, México. E-mail: delta_mt16@hotmail.com.
} 
cultural tourism route, who are the evaluators of the tourist route and finally where to start and finish the route according to the availability of time and money of the tourist.

Key words: Cultural Heritage tourism; Routes design; Competitiveness; South of Jalisco. 


\section{INTRODUCCIÓN}

De acuerdo con Alfonso Jiménez, la actividad turística en México como fuente generadora de ingresos en constante crecimiento, data de los años 1940, lo anterior, debido al impulso brindado por el entonces presidente de México Miguel Alemán. Durante su gobierno, continua Jiménez, se impulsó significativamente la creación de los primeros polos turísticos, como Acapulco, Manzanillo, Mazatlán, Puerto Vallarta, Cabo San Lucas, Cozumel, Isla Mujeres, Veracruz, Mérida, Guadalajara y la Ciudad de México. (JIMENEZ, 1990).

Uno de los avances más importantes fue la promulgación de la primera Ley Federal de Turismo, en 1949 (MCDONALD, 1970). En ese tiempo, la política relativa al turismo estaba a cargo de la Secretaría de Gobernación, y para proporcionarle mayor dinamismo se creó la Dirección General de Turismo, que posteriormente se convertiría en el Departamento de Estado de Turismo (JIMENEZ, 1990).

El turismo es una de las principales actividades económicas en el mundo, genera una importante derrama económica, inversión, empleos directos e indirectos, y puede contribuir al crecimiento económico y al progreso social de los países en vías de desarrollo, favorece la realización de actividades que atienden la demanda de los turistas como el hospedaje, la alimentación, el transporte, la diversión, el conocimiento de tradiciones, atractivos y de la cultura, entre otras. Además, atrae inversión nacional y extranjera e impulsa la infraestructura regional mediante la construcción de urbanización y vías de comunicación aérea, terrestre o marítima.

La investigación en el turismo se desenvuelve en diversas áreas, una de ellas es el turismo cultural, modalidad que se observa a su vez, desde diversos sectores con los cuales esta disciplina interactúa y que puede ir desde su relación con derechos humanos, desigualdad social, desarrollo sustentable, patrimonio, hasta producciones y políticas culturales. En ese sentido, este trabajo logra adentrarse a un aspecto que generalmente se toma como una acción sencilla pero exige una capacidad de integración y conjunción de aspectos sociales, económicos, políticos, empresariales y culturales por supuesto; la creación de Rutas de Turismo Cultural y su clara metodología para aplicar en diferentes regiones. 
El objetivo de este artículo es proponer una metodología para el diseño de rutas turísticas culturales utilizando la Región Sur de Jalisco como espacio geográfico para su diseño; el campo de estudio se delimita a diez municipios donde se analizan los atractivos turísticos culturales, la cantidad de establecimientos de servicios básicos y complementarios.

La importancia de la presente investigación radica en varios aspectos del quehacer turístico, del cual resalta como valor principal, la organización coherente, clara y con argumentos indispensables para la creación de una ruta de Turismo Cultural Regional que contribuyan a un adecuado recorrido por parte de los visitantes, de tal forma que al realizar actividades como parte de su viaje o excursión no se vean envueltos en la desinformación que pueda originar riesgos en la satisfacción tanto de necesidades básicas como lúdicas.

Más allá de la satisfacción a las personas que dentro de diversas posibilidades eligen visitar la Región Sur del Estado de Jalisco mediante servicios que den certeza a su visita, se recalca la gran importancia que el Turismo Cultural puede arrojar a la zona, de acuerdo con (PRADOS, 2001) los productos turísticos culturales están llamados a desempeñar un papel fundamental en el nuevo milenio. Se debe entender que esta actividad necesita alinearse a los principios éticos, sociales, ambientales, económicos y culturales como lo señala la Carta de Turismo Cultural del Instituto Nacional de Antropología e Historia (INAH, 2009), con la finalidad de contribuir a la valorización del patrimonio, la generación de empleos, factor decisivo para futuras inversiones y un papel importante en la cohesión social.

Es así que para la comunidad encargada de brindar servicios en esta región, principalmente hospedaje, alimentación y transporte así como Prestadores de Servicios Turísticos (PST) la investigación representa una firme oportunidad de crecer y desarrollarse, mostrando mayores signos de competitividad ante otros destinos ya consolidados con el fin de evidenciar la viabilidad que se tiene hacia futuras inversiones directas que permitan dinamizar la actividad económica regional.

Finalmente, el mayor sentido que tiene el realizar esta ruta es el de conglomerar el patrimonio cultural que posee la Región Sur para formar un producto turístico que permita a los visitantes poder conocer parte del significado de la vida del mexicano de ésta zona y bajo qué influjos se desarrolla la vida de manera cotidiana. 


\section{FUNDAMENTACIÓN TEÓRICA}

La Organización de las Naciones Unidas para la Educación, la Ciencia y la Cultura, conocida como la UNESCO, en su Conferencia Mundial sobre Patrimonio Cultural, celebrada en México en 1982, define al patrimonio cultural como:

En los pueblos, las obras de sus artistas, arquitectos, músicos, escritores y sabios, así como las creaciones anónimas, surgidas del alma popular, y el conjunto de valores que dan sentido a la vida, es decir, las obras materiales y no materiales que expresan la creatividad de ese pueblo; la lengua, los ritos, las creencias, los lugares y monumentos históricos, la literatura, las obras de arte y los archivos y bibliotecas (UNESCO, 1982).

De ahí el sentido, de la interpretación que hace la UNESCO sobre lo que para la humanidad debe significar el patrimonio cultural y que "debiera" tener influencia a nivel mundial, en todas las comunidades; así las naciones y las instituciones locales de cada país podrán trabajar conjunta y armónicamente en la principal labor para la cual se busca definir al patrimonio: Rescate, protección, preservación y divulgación.

En México, esta actividad fue legislada por el Gobierno Federal en 1972 (MAC DONALD, 1970). Fueron facultados El Instituto Nacional de Antropología e Historia (INAH), el Instituto Nacional de Bellas Artes (INBA) y la Secretaría de Educación Pública (SEP); dentro de las atribuciones que estas tres organizaciones tienen para beneficio de la cultura y las arte en el país, se encuentra la de crear asociaciones, comisiones, consejos y trabajar en conjunto con estos y a su vez con los tres diferentes órdenes de gobierno (Federal, Estatal y Municipal) para coadyuvar en todas las labores culturales y artísticas de interés nacional, así manifestado por la Ley Federal sobre Monumentos y Zonas Arqueológicos, Históricos y Artísticos. (CONACULTA, INAH. 2007)

Es el Manual Técnico del Consejo Nacional para la Cultura y las Artes (CONACULTA, INAH. 2007) en colaboración con el INAH quien acertada y asequible informa los tipos de patrimonio cultural que existen y la clasificación de éstos.

El patrimonio cultural tangible es "todo aquel elemento de una cultura que puede ser tocado, modificado, restaurado, reedificado o rescatado de la acción devastadora del tiempo y del hombre”. (UNIÓN, D. C. 2012) 
Esta clasificación basada en la Ley Federal sobre Monumentos y Zonas Arqueológicos, Artísticos e Históricos, comprende:

a) Patrimonio paleontológico. Incluye los restos fósiles de plantas y animales, cuyo origen se remonta a los tiempos en que aparecen los seres vivos sobre la tierra, hasta el fin del pleistoceno.

b) Patrimonio arqueológico. Está formado por la producción material que incluye: petroglifos, pintura mural, utensilios, la arquitectura - pirámides, montículos -, los entierros funerarios, la cerámica, la escultura, los códices y restos textiles, que provienen de grupos tan lejanos en el tiempo como los grupos de cazadoresrecolectores. Igualmente las producciones de los grupos que se encontraban en pleno auge al momento de la conquista, así como la flora y fauna aprovechadas por esos pueblos en su vida cotidiana.

c) Patrimonio histórico. Está formado por los bienes, muebles o inmuebles creados a partir del establecimiento del Virreinato (siglo XVI) hasta finales del siglo XIX. Incluye edificaciones religiosas, de gobierno y arquitectura civil; asimismo, caminos antiguos, reales de minas, misiones, cajas de agua, presidios o cárceles, cascos de haciendas y edificaciones de arquitectura tradicional, al igual que obras de arte producidas en ese periodo histórico.

Dentro del área considerada zona de monumentos históricos se conservan y protegen muebles e inmuebles, incluyendo edificios de carácter popular y contemporáneo, calles, plazas, mobiliario urbano y paisaje natural o casas habitación; todos estos elementos conforman la zona histórica y la imagen urbana de un centro de población. (UNIÓN, D. C. 2012)

Los documentos históricos también forman parte del patrimonio cultural e incluyen todo tipo de manuscritos, impresos, registros sonoros y visuales, producidos durante los periodos: Virreinal, de Independencia, Reforma, Porfiriano y Revolución Mexicana. (CONACULTA, INAH. 2007).

Algunos ejemplos de documentos históricos son: pleitos de tierra, títulos primordiales, autos judiciales, padrones, libros conventuales, actas de cofradía, crónicas y códices indígenas, actas inquisitoriales, leyes, decretos, declaratorias políticas, daguerrotipos, fotografías, grabaciones y documentales, que en su conjunto contienen el legado histórico de pueblos y comunidades de México. 
d) Patrimonio artístico. Lo constituyen aquellos bienes, muebles o inmuebles que revisten un valor estético relevante, atendiendo a cualquiera de las siguientes características: representatividad, inserción en determinada corriente estilística, grado de innovación, materiales y técnicas utilizadas y otras análogas. El patrimonio artístico incluye arquitectura, esculturas, pinturas, frescos, ornamentos, cerámica, muebles y textiles, una gran variedad de objetos realizados en lienzo, madera, vidrio, metales y papel, producidos en el siglo XX. Este tipo de patrimonio cultural está a cargo del Instituto Nacional de Bellas Artes y Literatura (INBA).

De acuerdo a la Ley Federal de Monumentos, una zona de monumentos artísticos es el área que comprende varios monumentos asociados entre sí, con espacios abiertos o elementos topográficos, cuyo conjunto revista valor estético en forma relevante. (UNIÓN, D. C. 2012).

e) Arquitectura relevante. Es la edificación de características arquitectónicas y antecedentes históricos únicos en la totalidad del conjunto. Su conservación y cuidado es determinante para la imagen urbana y su función en la ciudad suele generar actividades culturales y económicas que benefician a la población local y a los visitantes.

f) Arquitectura tradicional o de contexto. Edificación fundamentalmente del medio rural que corresponde a la imagen de poblados y comunidades de gran atractivo en zonas turísticas del país; se le encuentra en el entorno de zonas urbanas de transición entre el campo y la ciudad. Es testimonio de la cultura popular pues conserva materiales y sistemas constructivos regionales propios del medio, siendo de vital importancia su conservación como bien patrimonial.

A las anteriores categorías hay que agregar un denominador común: la arquitectura vernácula o popular, que al conservar el uso de procedimientos constructivos y materiales tradicionales, así como raíces formales y funcionales de regiones y zonas del país, constituyen un testimonio de enorme valor cultural, ya que por sus características denota un importante factor de la identidad cultural de una población o una región de la nación. (UNIÓN, D. C. 2012).

La arquitectura relevante, monumental o vernácula, tiene importancia igual como edificio aislado, que como parte de conjuntos y zonas o como entorno de otros monumentos. 
g) Patrimonio intangible. El patrimonio cultural intangible de acuerdo a la Ley Federal de Monumentos, "es la parte viva de un grupo que se expresa en sus cosmovisiones, usos y costumbres, rituales, música, habilidades artesanales e historia oral, entre otros innumerables rasgos de la cultura”. (UNIÓN, D. C. 2012).

Los cuentos, mitos y leyendas, surgen en un momento determinado de la historia y después son continuamente reelaboradas dando pauta para conocer las peculiaridades del pensamiento de los pueblos.

Tanto el patrimonio cultural tangible como el intangible constituyen la riqueza de los mexicanos, por lo que es un deber rescatarlo, conservarlo y difundirlo, ya que día con día resulta afectado por el crecimiento urbano desmedido y las migraciones del campo a la ciudad o al extranjero, y por consiguiente la transformación y/o pérdida de las identidades locales y regionales.

\section{PROCEDIMIENTO METODOLÓGICO}

La metodología que se utilizó para ésta investigación considera los siguientes elementos:

Se utilizó la técnica de investigación descriptiva, con el fin de buscar especificar las propiedades, las características y los perfiles importantes de personas, grupos, comunidades o cualquier otro fenómeno que se someta a un análisis.

Esta técnica permite analizar cómo es y cómo se manifiesta un fenómeno y sus componentes, por ello que se estudiaron las características sociales y económicas de los municipios previamente delimitados. Posteriormente se recurrió a recabar información que demarcó el espacio de estudio, esto propició conocer el entorno natural y social al que se arribó. Para ello se realizó una revisión documental, que a su vez coadyuvó para la elaboración del sustento teórico de la investigación y recopilación de los datos duros de carácter bibliográfico, hemerográfico y en línea (internet).

Una vez conocido esto, se procedió a identificar las diferencias entre las rutas y los demás espacios promotores del turismo. Se recurrió a los documentos afines a la actividad cultural de México y el mundo. Así fue como se inició el recabar de datos acerca del patrimonio cultural de los municipios y su posterior análisis para delimitar su 
viabilidad como atractivos turísticos. Aunado a lo anterior, se propusieron instrumentos para medir la disponibilidad y accesibilidad del patrimonio; los servicios adicionales al transporte, alimentación y hospedaje. Se dilucidaron aspectos necesarios para finalizar la elaboración de la ruta y se planteó una alternativa para transitar en ésta.

Finalmente, se aplicaron los pasos anteriores a los doce municipios mencionados.

\section{PRINCIPALES HALLAZGOS DE LA INVESTIGACIÓN Y /O PROPUESTA}

Con base en las investigaciones realizadas surgieron las siguientes recomendaciones y/ propuestas para el diseño de rutas turísticas culturales en el sur del Estado de Jalisco:

\subsection{UBICAR Y DELIMITAR EL TERRITORIO}

Se debe situar el campo de estudio y hacer las delimitaciones necesarias acorde al área donde se desea implementar la metodología propuesta en proporción al sector geográfico, social y económico que se pretende involucrar a la ruta.

Para el caso del Sur de Jalisco se eligieron doce municipios tentativos que para efectos de la planificación y promoción turística pertenecen a la integración de dos rutas ecoturísticas diseñadas por la Secretaría de Turismo Jalisco: "Ruta Ecoturística Sierra del Tigre" y "Ruta Ecoturística Sierra-Halo Volcanes".

La selección de los municipios se hizo con base en su empírica riqueza patrimonial tangible e intangible, que fuese capaz de sustentar la visita de turistas, excursionistas y viajeros que pretendan conocer dicha zona del Estado sin que esto represente una amenaza tanto para los destinos receptores ni para los visitantes, así mismo, ubicación geográfica (colindantes) y la ínfima distorsión de la desviación en su principal vía de comunicación (carretera) entre municipios.

Los municipios seleccionados integrados son: Atoyac, Sayula, Zapotlán el Grande, Tuxpan, Tecalitlán, Zapotiltic, Tamazula de Gordiano, Mazamitla, Valle de Juárez, Quitupan, La Manzanilla de la Paz y Concepción de Buenos Aires. 


\section{2 CLARIFICAR LAS CARACTERÍSTICAS DE LA RUTA}

Se tuvo presente qué elementos debería representar la ruta al visitante, en este caso, el patrimonio y posteriormente los atractivos culturales que poseen los municipios involucrados en la potencial ruta.

Se contempló que dichos elementos fueran complementados por una seria de servicios que se dirigieran a cubrir necesidades básicas del turista y/o excursionista. Finalmente se planteó que la gestión de la ruta fuera íntegramente representada por distintos sectores de la sociedad con la finalidad de que mayores actores sociales involucren en el proceso.

\subsection{IDENTIFICAR POTENCIALES ACTORES/PROMOTORES}

Se sugirió la identificación de actores primordiales para la elaboración y gestión de la ruta turística, específicamente, para promover la participación de todas las trincheras sociales productivas que pueden participar en la integración de la oferta. Para ello, se esbozó el planteamiento de (MERINERO RODRÍGUEZ \& ACOSTA ZAMORA, 2009), en el que se contempla la participación de: Administraciones públicas, empresas de servicios turísticos, grupos de interés empresarial, personas que poseen recursos turísticos, personas que generan opinión e influencia y Grupos sociales locales.

De este modo se manifestaron los segmentos sociales que pudiesen ser convocados para gestionar la ruta turística.

\subsection{DELIMITAR EL PATRIMONIO CULTURAL}

Se señaló la necesidad de conocer los conceptos que por patrimonio cultural se tiene en una instancia internacional rectora de la actividad cultural como lo representa la UNESCO. Posteriormente se propuso indagar en la conceptualización del patrimonio cultural a nivel nacional (para efectos de este trabajo: México) donde se plasman las características de dicho patrimonio y por ende su acotación respecto a otras 
manifestaciones. Esto se expresa claramente en la Ley Federal sobre Monumentos, Zonas Arqueológicos, Artísticos e Históricos (UNIÓN, 2012).

Una vez analizada esta información fue necesario esclarecer qué parte del patrimonio cultural se vería reflejado en una potencial ruta, en este caso, las manifestaciones culturales, el folklore y los museos, todo ello posible recolectar gracias a la clasificación de elementos del folklore propuesta por Boullón (1999) que fue aplicada de similar forma en los doce municipios.

\subsection{DISTINGUIR ENTRE EL PATRIMONIO CULTURAL Y EL ATRACTIVO TURÍSTICO CULTURAL}

Resulta evidente identificar aquellos elementos que conforman el patrimonio cultural de los pueblos utilizando cualquier método que resulte viable aunque son otros factores sociales o ambientales los que contribuyen a que la manifestación cultural sea considerada un atractivo turístico cultural.

\subsubsection{Factor disponibilidad.}

Es entendido que al hablar de disponibilidad en un atractivo turístico se encuadran diversos aspectos que van desde elementos como el tiempo o el espacio hasta la propiedad de dicho atracción; por lo cual, resulta preciso esclarecer qué componentes son los necesarios para que ese patrimonio mediante su análisis, pueda identificarse a su vez como un atractivo turístico.

Es por ello que se elabora un esquema donde se describe cuáles son los componentes necesarios para identificar si un atractivo turístico en realidad es disponible. 


\section{CUADRO 1 - DISPONIBILIDAD DEL PATRIMONIO}

\begin{tabular}{|c|l|l|l|l|l|c|}
\hline \multicolumn{2}{|c|}{ AGENTE } & \multicolumn{2}{c|}{ PATRIMONIO } \\
\hline \multirow{2}{*}{ Propiedad } & \multirow{2}{*}{ Grado conservación } & Horario & Acceso & $\begin{array}{c}\text { PTS o personal } \\
\text { apto para difundir }\end{array}$ & Tangible & Intangible \\
\hline Publica & Componentes & Diurno & Libre & Dueño & & $\mathrm{X}$ \\
\hline Privada & Espacios & Nocturno & Cuota & Encargado & $\mathrm{X}$ & \\
\hline & Volúmenes * & Mixto & & Guía Turistas & & \\
\hline & Materiales* & & & & & \\
\hline
\end{tabular}

Fuente: Elaboración propia (2011)

*solo para patrimonio tangible.

En este cuadro, se podrá identificar si un atractivo es disponible o no; primeramente, se debe comprender que este bosquejo califica las cualidades de un solo atractivo, por lo tanto, el primer factor a identificar será el patrimonio y con ello ubicarlo si tiene las características de tangible o intangible; posteriormente se traslada a resolver los agentes tales como propiedad, que puede ser pública o privada y lo que ello implica relativo al uso de suelo; el grado de conservación, que mide los niveles de alteración o describe la condición actual de componentes (elementos que llegan a complementar al patrimonio), de espacios (todo el entorno del patrimonio), volúmenes (las dimensiones físicas del patrimonio) y los materiales (componentes físicos que forman el patrimonio); el horario, que consta de identificar en qué momento del día puede se puede visitar o disponer del patrimonio y el personal profesional o aficionado que sea capaz de transmitir los valores culturales, estéticos y sociales.

\subsubsection{Factor accesibilidad}

Para calificar la accesibilidad del patrimonio también se propone un esquema que muestra los criterios a vislumbrar para deliberar si es accesible el patrimonio. 
CUADRO 2 - ACCESIBILIDAD DEL PATRIMONIO

\begin{tabular}{|c|l|l|c|c|}
\hline \multicolumn{2}{|c|}{ AGENTE } & \multicolumn{2}{c|}{ PATRIMONIO } \\
\hline Caminos & Transporte & Servicios & Tangible & Intangible \\
\hline Carretera Federal Libre & Terrestre & Gasolinería & $X$ & \\
\hline Carretera Federal Cuota & Marítimo & Taller Mecánico & & X \\
\hline Carretera Estatal & Aéreo & Central o terminal autobuses & & \\
\cline { 1 - 2 } Carretera Municipal & Fluvial & Baños & & \\
\cline { 1 - 2 } Otro tipo de camino & & $\begin{array}{c}\text { Renta de transporte motorizado y no } \\
\text { motorizado }\end{array}$ &
\end{tabular}

Fuente: Elaboración propia (2011).

En este esquema se identifica que los agentes determinantes para la accesibilidad del patrimonio son los "caminos" mediante los cuales se pueda acceder al sitio, que generalmente serán terrestres aunque teniendo en cuenta que para utilizar el transporte terrestre previamente se haya hecho uso del transporte marítimo, aéreo o fluvial; otra de las aristas es el 'transporte' que como se comenta, puede ser de los tipos anteriormente mencionados y que involucra toda clase de vehículos motorizados y no motorizados.

Cuando el patrimonio tiene las características de ser disponible es cuando éste presenta las peculiaridades de ser aprovechado con fines recreativos, presentar seguridad al visitante y existe la(s) persona(s) que pueden transmitir el valor y las particularidades que le hacen sobresalir; no es necesario que cumpla con todas las características que un esquema sugiera cumplir debido a que los entornos y propiedades de cada región del país o del mundo les hacen simplemente incomparables por más contraproducente que parezca el hecho de utilizar un esbozo.

La accesibilidad del patrimonio debe ser siempre encaminada a brindar la seguridad para el turista independientemente del medio de transporte que éste elija con la salvedad de que es uno de los principales motivos para que el turista se interne en un sitio que es ajeno al de su residencia. Cabe destacar que los accesos al patrimonio pueden, después de brindar la seguridad a los visitantes, incrementar su calidad tanto en caminos como en transporte; en el primer rubro gracias a las políticas turísticas que las autoridades puedan implementar y la segunda mediante la inversión de la iniciativa privada primordialmente.

Son estas propiedades las que consolidan al patrimonio como atractivo turístico, debido a que al acervo cultural y folklórico le comprenden elementos que comienzan a ser la base mínima para que se pueda ejercer la actividad turística; es así que se hace la 
delimitación entre el patrimonio cultural y el atractivo turístico cultural, la gran diferencia entre ser un sitio atractivo a ser un espacio con potencial vocación turística.

\subsection{SELECCIONAR ATRACTIVOS PARA LA RUTA Y ELEMENTOS REPRESENTATIVOS}

Una vez que el patrimonio o recurso turístico potencial ha sido diagnosticado como atractivo turístico, se debe trabajar en guiarle hacia la vocación turística a este activo que fue previamente identificado; hay que tener presente que ninguna manifestación cultural y/o artística es creada con tal proclividad de ser un atractivo (ANTON; FERNÁNDEZ; GONZÁLEZ, 2007), por ello se deben considerar factores de interacción entre población receptora y visitantes para no ver alteradas de forma negativa las costumbres y tradiciones.

Como elementos representativos se hace alusión a los personajes o las manifestaciones histórico-culturales de mayor significancia desde el ámbito internacional hasta local; ejemplo de ello, para este caso, sería involucrar nombres como José Luis Martínez, Juan Rulfo, Juan José Arreola, José Clemente Orozco, Mariachi Vargas de Tecalitlán, Hermanos Záizar, Luis Barragán, Rafael Urzúa o expresiones desde Pedro Páramo, Llano en Llamas, Portal Estilo Sayula, Bésame Mucho, La Feria, Confabularlo, Estaciones de Tren, Sonajeros, Paixtles, entre otros.

\subsection{DEFINIR LOS SERVICIOS A BRINDAR Y CAPACITACIÓN DE PRESTADORES DE SERVICIOS TURÍSTICOS}

En la actividad turística existen diversos servicios que son esenciales para la vida de este fenómeno, como actividad y como fenómeno social, de esos servicios, los básicos son: el hospedaje, la alimentación y el transporte.

Estas tres clases de servicios son las obligatorias mínimas para brindar al turista la seguridad que requiere al verse envuelto en un entorno social distinto al habitual; partiendo del cumplimiento de éstos se puede considerar un destino apto en servicios para la actividad turística. 


\subsubsection{Otros servicios relevantes.}

Existen otros servicios adicionales que auxilian complementariamente al turista al momento de llegar a un destino distinto a su lugar de residencia (BOULLÓN, 1999).

Agencias de viajes, información turística, guías de turistas, cambios de moneda, dotación para congresos y convenciones, transportes turísticos, primeros auxilios, guarderías, estacionamientos, farmacias, hospitales, cuerpo policiaco.

Un cuadro para recoger los datos referentes a la existencia de dichos servicios puede ser:

CUADRO 3 - VERIFICACIÓN SERVICIOS EN EXISTENCIA

\begin{tabular}{|l|l|l|l|}
\hline Servicios & Posee & No posee & Cantidad \\
\hline Alimentación & X & & 5 \\
\hline Transporte & & & \\
\hline Hospedaje & & & \\
\hline Agencias de viajes & & & \\
\hline Información turística & & & \\
\hline Guías de turistas & & & \\
\hline Cambios de moneda & & & \\
\hline $\begin{array}{l}\text { Dotación para congresos } \\
\text { convenciones }\end{array}$ & & & \\
\hline Transportes turísticos & & & \\
\hline Primeros auxilios & & & \\
\hline Guarderías & & & \\
\hline Estacionamientos & & & \\
\hline Farmacias & & & \\
\hline Hospitales & & & \\
\hline Cuerpo policiaco & & & \\
\hline Fuente: BOULLON (1999). & & & \\
\hline
\end{tabular}

Una vez que se han verificado los servicios que disponen los sitios que integrarán la ruta, se procede a la identificación personalizada de cada uno de los establecimientos con la finalidad de gestionar su participación en el proyecto de la ruta y clarificar todos los lineamientos que habrá de considerar y cumplir para sumar al resto de servicios que se pretenden ofrecer.

La finalidad de esto es poder cimentar las bases para que en el futuro se analice la formación de un cluster turístico que en acuerdo a las expectativas del visitante, pueda brindar los servicios de forma profesional, lo que ocasiona mayores dividendos para el desarrollo regional (ESPINOZA, 2010). 
4.7.2 Capacitación de los prestadores de servicios turísticos.

La preparación de los prestadores es fundamental para que la ruta pueda funcionar, debido a que es ésta la gente que dinamiza la actividad turística; de su capacidad depende en gran medida que el visitante goce de su estancia y genere la satisfacción que el turista desea, con las implicaciones positivas que esto refleja.

Algunos posibles cursos sugeridos a impartir ante los distintos prestadores de servicios son:

* Introducción al turismo cultural.

* Formulación y Evaluación de Proyectos

* Atención y Servicio al cliente.

* Software aplicado

* Principios de Contabilidad.

* Estrategias de marketing.

Si bien estos cursos no son los únicos, sí brindan una base y panorama general de los aspectos que se deben solventar como primera necesidad para que el servicio a brindar, además de satisfacer, pueda ofrecerse con criterios de calidad.

\subsection{DISEÑAR Y UNIFORMAR LA SEÑALÉTICA}

La señalética siempre ha sido un tema controversial en el turismo; los primeros esfuerzos para homogeneizar las señales de uso turístico se dio en Manila en 1980 (LANQUAR, 2008). Respecto a los símbolos a utilizar para representar las diversas manifestaciones culturales se debe debatir sobre la imagen que representará dichas actividades con la finalidad de difundir su uso y estandarización en todo el territorio. El mismo Dr. Lanquar propone el siguiente esquema, al que llama: 
- Promoción

- Publicidad.

- Señalización

MOSTRAR Y ATRAER

\section{INFORMAR Y ANIMAR}

Promoción.

- Folletos.

- Puntos de información.

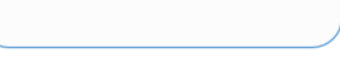

- Indicadores.

- Info-stations.

- Marcas.

- Localizadores.

- Itinerarios.

- Puntos de información.

FUENTE: LANQUAR, 2008.

El esquema expone en tres partes el deber ser de la señalización: "Mostrar y atraer", "Informar y Animar” y “Guiar y Recibir". También distingue los elementos que habrá de cumplir la señalización para acatar los tres principios expuestos.

Son tres los elementos en que se propone aplicar estos principios: Atractivos, Servicios, Distancias y sitios.

Finalmente, se debe gestionar con los involucrados en la elaboración de la ruta para homogeneizar y plasmar la actitud o vocación de la ruta turística; esto permitirá que genere un sello distintivo respecto otros atractivos. Primordial para que quede "marcado" en el visitante y haga la promoción "de boca en boca" que complementa las estrategias mercadológicas.

\subsection{APROVECHAR LAS ESTRATEGIAS MERCADOLÓGICAS PARA POSICIONAR LA RUTA}

Para planear y seguir las directrices marcadas es necesario elaborar un análisis para su comercialización. En el presente trabajo se proponen algunas estrategias elaboradas por J. Bigné, Xavier Font y Luisa Andreu (2000). 
CUADRO 4 - ELEMENTOS DEL MARKETING ESTRATÉGICO

\begin{tabular}{|l|l|}
\hline $\begin{array}{l}\text { Análisis del entorno genérico de } \\
\text { carácter social, } \\
\text { tecnológico y político. }\end{array}$ & $\begin{array}{l}\text { Este contexto marca cual herramientas los mecanismos para } \\
\text { consolidar la planeación de la ruta como producto turístico. }\end{array}$ \\
\hline $\begin{array}{l}\text { Definición del mercado de referencia. } \\
\text { Segmentación del mercado. }\end{array}$ & $\begin{array}{l}\text { Los trabajos deben ser encaminados a la satisfacción de las } \\
\text { necesidades de la demanda. }\end{array}$ \\
\hline Análisis de competitividad. & $\begin{array}{l}\text { Fraccionar la demanda por características afines y brindar } \\
\text { opciones de goce diversas del mismo producto. }\end{array}$ \\
\hline Megamarketing. & $\begin{array}{l}\text { Distinguir las características de la ruta como producto y } \\
\text { acondicionarla para las exigencias de la competitividad actual. }\end{array}$ \\
\hline $\begin{array}{l}\text { Alianzas estratégicas } \\
\text { proveedores y competidores. }\end{array}$ & $\begin{array}{l}\text { Identificar los sectores a persuadir para ingresar a los mercados } \\
\text { y promover el producto con las personas que representan } \\
\text { injerencia popular (relaciones públicas). }\end{array}$ \\
\hline $\begin{array}{l}\text { Aprovechar las ventajas de las } \\
\text { nuevas tecnologías. }\end{array}$ & $\begin{array}{l}\text { Ayuda a consolidar un producto mega-diverso tanto en } \\
\text { afluencia de turistas como en servicios proporcionados y su } \\
\text { alcance. }\end{array}$ \\
\hline $\begin{array}{l}\text { Ir en armonía con todas las manifestaciones de comunicación } \\
\text { que surgen y se arraigan en la sociedad con la finalidad de } \\
\text { atraer y fascinar al potencial turista. }\end{array}$ \\
\hline
\end{tabular}

Fuente: Marketing de Destinos Turísticos: Análisis y Estrategias de Desarrollo ${ }^{1}$

\subsection{ESTABLECER EL CONSEJO EVALUADOR DE LA RUTA}

Así como pueden ser promotores en la elaboración de la ruta, las administraciones públicas de todos los órdenes de gobierno, la empresas de servicios turísticos, los grupos de interés empresarial, las personas que poseen recursos turísticos, las que generan opinión e influencia, y los grupos sociales locales, en conjunto deben ser quienes constituyan una entidad (inclusive la misma que promovió la ruta) que tenga las debidas atribuciones para valorar y medir el cumplimiento de metas y objetivos.

Otra alternativa es que la agrupación promotora derive la facultad de evaluar y medir, a otro colectivo que tenga la capacidad de poder generar una labor objetiva e imparcialmente, que de forma profesional sea capaz de transmitir resultados que permita a los tomadores de decisiones, ejercer el mejor curso de acción para el proyecto.

Las metas que llevarán al cumplimiento de los objetivos se pueden catalogar, para su evaluación y medición, en estos rubros ${ }^{2}$ : Financiero, técnico, operativo, social y turístico.

La evaluación turística analiza y mide de forma cualitativa la vocación turística manifestada en los nuevos prestadores de servicios turísticos, así mismo, de forma

\footnotetext{
${ }^{1}$ Cuadro basado en las Funciones del Marketing Estratégico (Bigné, Font \& Andreu, 2000).

${ }^{2}$ Es irrelevante el orden del título de éstos contenidos.
} 
cuantitativa, el contraste de los indicadores relacionados con afluencia de turistas y excursionistas, periodos de estancia, gasto promedio, preferencia de actividades.

\subsection{SUGERIR ALTERNATIVAS PARA RECORRER LA RUTA ACORDE AL TIEMPO Y DINERO}

Uno de los dilemas que encuentra el visitante sea como turista o excursionista cuando no viaja bajo la tutela de algún prestador de servicios turístico afín (guía turístico) es que desconoce cómo puede hacer rendir eficientemente el dinero y el tiempo disponibles. Es por ello que se proponen algunas estrategias que pueden hacer más llevadera la estancia en una ruta de turismo cultural basado en los dos factores antes mencionados que en el turismo denotan clara relación.

\section{CONCLUSIONES}

El turismo cultural es un hecho evidente en la gran parte de México como país, en el caso del estado de Jalisco, la tendencia hacia las visitas públicas por concepto de cultura ha ido incrementando, sobre todo con la distinción de 5 pueblos mágicos distribuidos en el estado. En el sur de Jalisco a parte de los dos pueblos mágicos de Tapalpa y Mazamitla, históricamente, la Región ha sido culturalmente activa como lo muestran sus diversas manifestaciones artístico-culturales, así como los creadores nativos de esta región.

Teniendo en cuenta lo anterior, pese a que en la región se cuenta con los diversos recursos ya mencionados, es necesario, para su buen aprovechamiento turístico, tomar en cuenta las siguientes recomendaciones:

- La actividad turística planeada e implementada bajo las premisas del desarrollo regional, puede incidir en la disminución de la migración.

- Sea una ruta, un centro, una zona o un corredor turístico, lo fundamental es generar organismos e instrumentos de evaluación para que la actividad turística prolifere al corto, mediano y largo plazo. 
- La infraestructura en vías de comunicación es suficiente para diseñar una ruta de turismo cultural; los servicios obligados (alimentación, hospedaje y transporte) son ampliamente satisfechos por la cantidad de establecimientos en tres puntos (Sayula, Zapotlán el Grande y Mazamitla), aunque se brindan éstos servicios en los doce puntos de la ruta.

- El desarrollo de la ruta de turismo cultural debe gestarse integralmente por gobiernos, sociedad (grupos sociales) e iniciativa privada, pues la armonía entre todos los actores culminará en exponer una ruta que sea interesante y competitiva.

- Las rutas "Sierra del Tigre" y "Sierra Halo-Volcanes" (turismo de naturaleza) implementadas por la Secretaría de Turismo del Estado de Jalisco se pueden ver complementadas por una ruta de turismo cultural que sea integrada por municipios de cada una de éstas.

- Son necesarias las tecnologías de la información (TIC's) para hacer adecuada comercialización de la ruta cultural y expandirla a toda clase de mercados a través de la virtualidad.

- Es recomendable estudiar los fenómenos socioeconómicos y naturales que en el tiempo y el espacio se manifiestan. Un ejemplo de ello sería el brote de violencia que ha venido viviendo la región, o las crisis económicas y los cambios en el entorno natural que de determinada manera influyen en el quehacer turístico.

- Una propuesta es eso, brindar una opción al interesado para que pueda aplicar lo aquí manifestado o valorar lo que para éste resulte valorable y que pueda complementar ideas ajenas o desconocidas.

\section{REFERENCIAS}

ANTON, S. C.; FERNÁNDEZ, A. T.; GONZÁLEZ, F. R. Los Lugares Turísticos. En: A Propósito del Turismo. La Construcción Social del Espacio Turístico ANTON, S. C.; GONZÁLEZ, F. R. (coords.). Barcelona: UOC. 2007. p. 103-193.

BIGNÉ, J.; FONT, X. ; ANDREU, L. Marketing Estratégico en Destinos Turísticos. Madrid: ESIC EDITORIAL, 2000.

BOUllon, R. C. Planificación del Espacio Turístico. Ciudad de México: Trillas, 1999. 
ESPINOZA, S. En Ciernes, el Primer Cluster Turístico. Alcaldes de México. v. 8, p. 78-80., agosto de 2010.

INAH. Carta Turismo Cultural. Disponible en: http://www.antropologia.inah.gob.mx/pdf/pdf_marcos/CARTA\%20TURISMO\%20CU LTURAL. Consultado el: 28/04/2013

JIMÉNEZ, M. A. Turismo Estructura y Desarrollo. Ciudad de México: Interamericana, 1990.

LANQUAR, R. Señalización Turística Excelencia Obligada. Documento presentado en $2^{\circ}$ CONGRESO NACIONAL DE PLANIFICACIÓN, DINAMIZACIÓN Y CALIDAD EN LOS SERVICIOS TURÍSTICOS, 2010. Córdoba, España.

MACDONALD, E. Una aproximación al conocimiento turístico. México: F.C.P. y C. UNAM, 1970.

MERINERO RODRÍGUEZ, R.; ZAMORA ACOSTA, E. La Colaboración entre los Actores Turísticos en Ciudades Patrimoniales. Reflexiones para el Análisis del Desarrollo Turístico. PASOS, Revista de Turismo y Patrimonio Cultural, v. 7, n. 2, p. 219-238, 2009.

PRADOS, E. P. Turismo Cultural: un Segmento Turístico en Expansión. En CONGRESO VIRTUAL DE TURISMO CULTURAL, 2010. Argentina. Disponible en: http://www.naya.org.ar/turismo/congreso/ponencias/elisa_prados.htm. Consultado el: $15 / 04 / 2013$

UNESCO. Disponible en: http://www.unesco.org/new/es/mexico/workareas/culture/outline/. Consultado el: 18/04/2013.

UNIÓN, D.C. Ley Federal sobre Monumentos y Zonas Arqueológicos, Artísticos e Históricos. Disponible en: http://www.diputados.gob.mx/LeyesBiblio/pdf/131.pdf. Consultado el: 10/04/2013

Recebido em: 15-11-2012

Aprovado em: 15-12-2012 